\title{
Die chinesischen Wirtschaftsreformen seit 1978 unter besonderer Berücksichtigung der Sonderwirtschaftszonen
}

\author{
Von Martin Klingst
}

\section{Einleitung}

Seit mindestens drei Jahren wird in den westlichen Medien vermehrt über eine neue Wirtschaftspolitik der Volksrepublik China berichtet. Diese wird dabei zumeist als "Rückkehr China's zum Kapitalismus", "China's Weg zur Marktwirtschaft" oder als "China's Abkehr vom Marxismus" bezeichnet.

Ohne diese Formulierungen sogleich in den Bereich des rein Floskelhaften rücken oder sie dem reinen Wunschdenken einiger Wirtschaftskreise zuordnen zu wollen, überzeichnen sie nicht nur Ausmaß und Auswirkungen der chinesischen Wirtschaftsreformen, sondern interpretieren diese auch nur unzulänglich, wenn nicht sogar falsch.

Es soll hier versucht werden, die chinesischen Wirtschaftsreformen in ihren sowohl ökonomischen als auch rechtlichen und politischen Zusammenhängen darzustellen. Die Ausführungen werden sich größtenteils an den in der Volksrepublik seit 1980 eingerichteten Sonderwirtschaftszonen orientieren, dies aber nicht nur einer konzentrierteren Darstellung wegen, sondern gerade deshalb, weil sich die Gemüter vornehmlich an der Einrichtung dieser "Zonen des reinen Kapitalismus" erregt haben.

Bevor hierauf im einzelnen einzugehen ist, soll zu einem besseren Gesamtverständnis der Vorgänge die Entwicklung der chinesischen Wirtschaft in diesem Jahrhundert in groben Zügen dargestellt werden.

\section{Die Entwicklung der chinesischen Wirtschaft im 20. Jahrhundert}

Vor 1949 war die industrielle Produktion Chinas im Verhältnis zu seiner Größe und seinem Bevölkerungsreichtum relativ unbedeutend und konzentrierte sich vornehmlich auf die nordöstlichen Provinzen sowie die großen Küstenstädte. Erschwerend für eine wirtschaftliche Entwicklung war, daß gerade die produktionsintensiven Gebiete - wie z. B. Shanghai - unter ausländischer Vorherrschaft standen, Profite abgeschöpft und ins Ausland transferiert wurden und der Handel größtenteils zwischen diesen chinesischen "Vertragsgebieten" und dem Ausland ohne Einbeziehung (des "restlichen") Chinas stattfand.

In der ersten Hälfte des 20. Jahrhunderts stellten noch ca. 3/4 der Bevölkerung ihre Arbeitskraft der Landwirtschaft und den hiermit in Zusammenhang stehenden Wirt- 
schaftszweigen zur Verfügung und bestritt dieser Wirtschaftssektor ca. 2/3 des volkswirtschaftlichen Nettoeinkommens. ${ }^{1}$

Aber selbst der Landwirtschaftssektor konnte kaum als produktionsintensiv bezeichnet werden. Sowohl ständige kriegerische Auseinandersetzungen als auch die Eigentumsverhältnisse an Grund und Boden hemmten eine effiziente Nutzung der bebaubaren Flächen. So kontrollierten $12 \%$ der Landeigentümer ungefähr die Hälfte der kultivierten Flächen, während ca. $60 \%$ der Bevölkerung nur $24 \%$ des landwirtschaftlich nutzbaren Bodens besaßen. ${ }^{2}$ Nach dem Erfolg der Revolution im Jahre 1949 sah sich die Volksrepublik daher einer fast unübersehbaren Vielzahl wirtschaftlicher Probleme gegenüber. Wegen der Vorrangigkeit des Landwirtschaftssektors für die Volksversorgung befaßte sich die neue Führung in den ersten Jahren daher auch vornehmlich mit einer umfassenden Landreform.

Kennzeichnend für den "neuen Weg" nach der siegreichen Revolution war die starke Abschottung der Volksrepublik gegenüber jeglichen Auslandsbeziehungen - vor allem aber gegenüber dem westlichen Ausland sowie Japan - und das Bekenntnis zum Prinzip der "self-reliance". Ursache hierfür war die frühere starke Abhängigkeit der chinesischen Wirtschaft vom Ausland und die "Überfremdung" des eigenen Marktes.

Trotz dieser rigiden Abschottung nach außen, herrschte nach innen - und hier insbesondere im Industriebereich - vorerst noch eine gewisse Liberalität, zumal die neue Führung der Kenntnisse und Fähigkeiten der chinesischen Unternehmer und Manager aus "kapitalistischen Zeiten" bedurfte. Die zunehmende Verstaatlichung ganzer Produktionszweige führte aber ab Mitte der 50er Jahre zu einer verstärkten zentralstaatlichen Wirtschaftsplanung und -verwaltung und letztendlich zur Anlehnung an das zentralistische Wirtschaftsmodell der Sowjetunion.

Wies der erste Fünfjahresplan von 1953-1957 noch beträchtliche Erfolge auf, so wurde der zweite von 1958-1962, mit dem der entscheidende Sprung nach vorne gemacht werden sollte, zu einem vollständigen Mißerfolg. Das schlechte Ergebnis führte erneut zu einer Veränderung der Wirtschaftspolitik, vor allem in der Prioritätensetzung. ${ }^{3}$ Nahm die Schwerindustrie bislang den ersten Platz in der Rangfolge wirtschaftlicher Ziele ein, fiel sie nunmehr auf Platz drei zurück, während die Landwirtschaft auf Platz eins vorrückte und die Leichtindustrie Platz zwei einnahm. Auch wurden in begrenztem Maße und unter staatlicher Aufsicht sowohl in der Landwirtschaft als auch in der Industrie zu Zwecken der Produktionssteigerung wieder private Wirtschaftsaktivitäten zugelassen. ${ }^{4}$

Diese neue Politik kam aber nur bedingt zum Zuge und war von ständigen innerparteilichen Machtkämpfen und Diskussionen um die "richtige Linie" begleitet. Der dritte 1965 in Planung gegebene Fünfjahresplan von 1966-1970 konnte infolge der im Jahre 1966
(5) S. 42.
(5) S. 41.
(12) S. 1
Ebd. 
einsetzenden Kulturrevolution nie realisiert werden. ${ }^{5}$ In den folgenden Jahren wurden weite Teile der Führungskräfte sowie viele Arbeiter und Angestellte zur Arbeit aufs Land geschickt und die staatlichen Wirtschaftsplanungsinstitutionen in ihrem Personalbestand auf ein Minimum reduziert. ${ }^{6}$ Die zunehmenden außenpolitischen Konflikte mit der UdSSR sowie die starken innenpolitischen Auseinandersetzungen im Rahmen der Kulturrevolution führten in weiten Bereichen der Wirtschaft zu einem Stillstand und zu einer noch stärkeren Isolation Chinas von der restlichen Welt. Alle Vorschläge in Richtung einer Offnung der Volksrepublik galten als antisozialistisch und wurden meist als Landesverrat geahndet. ${ }^{7}$

Erst nach dem Ende der Kulturrevolution und dem Tode Maos im September 1976 entflammten wieder Diskussionen über eine verantwortungsvolle Wirtschaftsplanung. Nachdem die "Viererbande" mit und um Maos Witwe 1978 ausgeschaltet wurde und sich die Erbfolge zugunsten der Reformer abzeichnete, wuchs die öffentliche Kritik an der übermächtigen und inkompetenten zentralistischen Verwaltung, einer überalterten und rückständigen Industrie und Technologie sowie an einer ineffizienten staatlichen Wirtschaftsplanung. ${ }^{8} \mathrm{Zu}$ Anfang geriet vor allem die Landwirtschaft in das Kreuzfeuer der Kritik. Hatte sich doch das Volkskommunensystem als unfähig erwiesen, sowohl genügend Nahrungsmittel für eine ständig wachsende Bevölkerung zu produzieren als auch für eine angemessene Verteilung der vorhandenen Nahrungsmittel zu sorgen. So erlagen noch Ende der 50er Jahre schätzungsweise mehrere Millionen Chinesen dem Hungertod $^{9}$ und hatten in den 70er Jahren immer noch zwischen 100 und 200 Millionen Chinesen nicht ausreichend zu essen. ${ }^{10}$

\section{Grundzïge der chinesischen Wirtschaftsref ormen}

Die größten Veränderungen fanden aus o. a. Gründen daher auch im Bereich der Landwirtschaft mit der Einführung eines vertraglichen Verantwortlichkeitssystems statt, das den Haushalt als neue Produktionsbasis vorsieht.

Hierbei handelt es sich um eine umfassende Reformierung des ländlichen Kollektivsystems. ${ }^{11}$ Der Volkskommune wurde die Administrationsbefugnis entzogen und diese den Gemeindeverwaltungen übertragen, so daß Verwaltungs- und Produktionsebenen (das Dorf bildet die Produktionsbrigade) identisch sind und das früher vorherrschende Herrschaftsverhältnis zwischen der übergeordneten Volkskommune und den untergeordneten

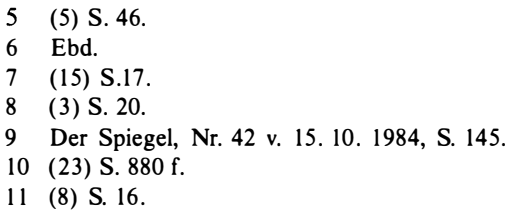


Dörfern als Produktionsbrigaden aufgehoben ist. ${ }^{12}$ Auf diese Weise gelang es, Verantwortung auf die untere Produktionsebene zu verlagern und die einzelnen Dörfer zu größerer Produktivität zu animieren.

Darüberhinaus können Gruppen von Bauern, einzelne Familien (Haushalte) oder sogar Einzelpersonen mit den Volkskommunen, die weiterhin (staatlicher) Eigentümer von Grund und Boden sind (vgl. Art. 10 der Verfassung vom 4. Dezember 1982), ${ }^{13}$ Verträge abschließen, mittels derer - bei Beibehaltung des sozialistischen Gemeineigentums an Grund und Boden - den Bauern für eine bestimmte und vertraglich festgelegte Zeit Land zur eigenen Bewirtschaftung zur Verfügung gestellt wird. ${ }^{14}$

In den zwischen den Volkskommunen und den "Haushalten" abgeschlossenen Verträgen werden in der Regel die Größe der zu bewirtschaftenden Flächen, Höhe und Art der von den "Haushalten" an den Staat (i. e. Volkskommune) abzulief ernden Erträge sowie die von ihnen zu entrichtenden Steuern und Beiträge zu den Wohlfahrts- und Investitionsfonds der Volkskommunen festgelegt. ${ }^{15}$ Die Erträge, die der "Haushalt " über das Produktionssoll hinaus erwirtschaftet, dürfen von diesem für die eigene Kasse auf den freien Märkten verkauft werden bzw. verpflichtet sich der Staat, diese zu Prämienpreisen abzukaufen. ${ }^{16}$

Angespornt von den ersten Erfolgen der Landwirtschaftsreform, zollte die neue Führung nun auch dem industriellen Sektor verstärkte Aufmerksamkeit und prägte den Begriff der "Vier Modernisierungen "17 (Landwirtschaft, Industrie, Verteidigung, Wissenschaft und Technologie). Die Produktionsprioritäten wurden zugunsten von Konsumartikeln verändert, Entscheidungsbefugnisse von der zentralstaatlichen Wirtschaftsverwaltung in Beijing auf regionale und lokale Entscheidungsgremien bzw. auf die Unternehmen selbst verlagert, um hierdurch die Produktion bedürfnisgerechter und effizienter gestalten zu können. Die reine staatliche Gewinnabschöpfung wurde größtenteils eingestellt und den Unternehmen gestattet, den Gewinn nach Abzug von Steuern und Abgaben einzubehalten und über dessen Verwendung in einem bestimmten Rahmen selbst zu befinden. Die Unternehmen dürfen zusätzlich zum garantierten Grundgehalt ein Prämiensystem einführen, um somit den Arbeitern und Angestellten einen Leistungsanreiz zu bieten. Der Staat hat außerdem das Recht, unproduktive Unternehmen zu schließen sowie ineffizientes Management abzusetzen. Den Arbeitnehmern wurde hinsichtlich der Besetzung des Unternehmensmanagements ein erweitertes Mitspracherecht eingeräumt.

Hand in Hand mit diesen Wirtschaftsreformen ging die Offnung des chinesischen Marktes nach außen. Schon auf der 3. Plenartagung des XI. Zentralkomitees der KP Chinas im Jahre 1978 wurde die Offnung Chinas nach außen als Prinzip des sozialistischen Aufbaus bezeichnet. ${ }^{18}$

12 (8) S. 17

13 Quelle: Review of Socialist Law, Vol. 9 No. 2, 1983, S. $184 \mathrm{ff}$.

14 (8) S. 16.

15 (22) S. 9.

16 (8) S. 17

17 (6) und (2) S. $11 \mathrm{f}$.

18 (15) S. 18 
Grund für die Offnung waren sowohl innere als auch äußere Faktoren. Zum einen war die chinesische Wirtschaft gekennzeichnet von ineffizienten industriellen und administrativen Strukturen, einem Mangel an gut ausgebildeten Fachkräften sowie von schlechten Infrastrukturen und einer veralteten Technologie. ${ }^{19}$ Hinzu kam ein wachsendes Konsumbedürfnis der chinesischen Bevölkerung, das weder qualitativ noch quantitativ befriedigt werden konnte. ${ }^{20}$ Zum anderen verlangte die Entwicklung der Volkswirtschaft und ihre Anpassung an den Weltmarkt die Kenntnis bereits anderswo existierender Produktionsweisen und Technologien und somit zwangsweise eine Offnung des eigenen Marktes für ausländische Einflüsse. Der chinesische Wirtschaftswissenschaftler Chen Qiwei beschreibt die Notwendigkeit einer Offnung der Volksrepublik mit der um sich greifenden Erkenntnis, daß Konkurrenz auf dem internationalen Markt der stärkste Stimulator für die Steigerung der eigenen Produktivkräfte sei und der Außenhandel in den meisten Staaten einen immer gewichtigeren Beitrag zum Bruttosozialprodukt leiste. ${ }^{21}$

Mit aus diesen Gründen entschloß sich die Volksrepublik Ende der 70er Jahre, Sonderwirtschaftszonen (SWZ) einzurichten, die das Reich der Mitte für ausländische Investoren attraktiver machen sollten.

\section{Die chinesischen Sonderwirtschaftszonen}

Die vier ersten SWZ Shenzhen, Zhuhai und Shantou in der Provinz Guangdong sowie Xiamen in der Provinz Fujian befinden sich entlang der chinesischen Küste. Zu dieser Standortwahl haben sicherlich sowohl die traditionelle Ausrichtung dieser Gebiete nach Ubersee als auch die Tatsache, daß eine Vielzahl liquider Auslandschinesen aus diesen Regionen stammt, auf deren wirtschaftliches Engagement die Chinesen vor allem setzen, als auch die unmittelbare Nachbarschaft von Shenzhen zu Hongkong und von Zhuhai zu Macao eine entscheidende Rolle gespielt. ${ }^{22}$

Zusätzlich zu diesen 4 SWZ wurde 1984 die "Offnung" von weiteren vierzehn Küstenstädten beschlossen, die z. T. das Recht verliehen bekommen haben, ihrerseits SWZ einzurichten. ${ }^{23}$ Diese designierten SWZ-Standorte verteilen sich über die gesamte Küste, nämlich von der südlichen Autonomen Region Guangxi Zhuang bis hin zur nordöstlichen Provinz Liaoning. ${ }^{24}$

Bevor die für Ausländer in den SWZ geltenden Investitionsbedingungen im einzelnen dargestellt werden, bedarf es zuerst einer grundsätzlichen Betrachtung des Phänomens "Sonderwirtschaftszone".

19 (3) S. 23.

20 (8) S. 16.

21 (8) S. $22 \mathrm{f}$.

22 (17) S. 166.

23 Neue Züricher Zeitung vom 10. 4. 1984.

24 Ebd. 


\section{Das Phänomen "Sonderwirtschaftszone"}

Allgemein geläufig sind Begriffe wie "Zollfreie Zonen", "Freihäfen", "Freihandelszonen". In der Bundesrepublik kennen wir vor allem die Frei(handels)häfen, die vornehmlich als Umschlagplatz für Waren aus aller Herren Länder fungieren, ohne daß jeweils Import- und Exportzölle fällig werden, sowie die sog. duty-free-shops, wo der Verkauf von Einzelwaren ohne Zollaufschlag an Auslandsreisende gestattet ist. In diesen "Freien Wirtschaftszonen" ist aber nur eine geringe wirtschaftliche Aktivität erlaubt. Demgegenüber stehen z. B. die sog. "Export(waren)-Verarbeitungszonen" mit einer Fülle wirtschaftlicher Betätigungen. Heute gibt es mehr als 200 Freizonen für Handel und Fertigung und mindestens $15 \%$ des Welthandelsvolumens werden dort produziert oder umgeschlagen. ${ }^{25}$

Eine der bekanntesten europäischen Freizonen befindet sich am internationalen Flughafen in Shannon, Irland. Ihre Einrichtung galt ehedem als Initialzündung für die Industrialisierung Irlands. ${ }^{26}$ Die bekanntesten außereuropäischen Freizonen befinden sich in Hongkong, Singapur u. vielleicht Macao. Gerade in den sich entwickelnden Ländern des asiatischen Raums trifft man häufig auf Freizonen. ${ }^{27}$ So verfügen $u$. a. auch Taiwan, die Philippinen, Pakistan, Thailand, Malaysia, Südkorea, Indonesien, Indien, Sri Lanka und nun auch die VR China über Freie Wirtschaftszonen.

Obwohl sich die Freizonen sowohl in ihrer jeweiligen geographischen Ausdehnung als auch in ihren Aktivitäten z. T. erheblich voneinander unterscheiden, handelt es sich bei den Freizonen in den sich entwickelnden Ländern zumeist um sog. Export(waren)Verarbeitungszonen bzw. Export(waren)-Produktionszonen.

Hier liefert der jeweilige Territorialstaat den ausländischen Investoren in der Hauptsache günstige Rahmenbedingungen, wie billige Arbeitskräfte, Produktionsmittel und Rohstoffe sowie vorteilhafte Steuer-, Zoll-, Arbeits- und Devisenbestimmungen. Die ausländischen Investoren stellen Kapital und ihr technologisches know-how zur Verfügung. Von der Grundkonzeption her erhofften sich die SWZ-Länder hiervon positive Auswirkungen auf die Infrastruktur, die Entwicklung eigener Industriezweige und den Arbeitsmarkt sowie letztendlich auf den Aufbau einer eigenen florierenden Exportindustrie. ${ }^{28}$ Dies hat sich bislang jedoch nur in Hongkong, Singapur, z. T. in Südkorea, Taiwan und vielleicht Malaysia bewahrheitet. In den vielen anderen Ländern ist die von der Einrichtung einer SWZ erhoffte Initialzündung für einen allgemeinen wirtschaftlichen Aufschwung bislang ausgeblieben, ja wirken sich die SWZ in vieler Hinsicht nicht nur hemmend sondern z. T. sogar kontraproduktiv für die soziale und ökonomische Entwicklung aus. Ursachen hierfür sind $u$. a. - verkürzt dargestellt - sowohl die historischen, politischen, sozialen und ökonomischen Bedingungen in den sich entwickelnden

\footnotetext{
(11) zitiert Michael van Notten.

(18) S. 70.

27 (20) Zur Entwicklung der Wirtschaftssonderzonen im asiatischen Raum vgl. S. 205.

28 (18) S. $69 \mathrm{ff}$.
} 
Ländern als auch das geringe Interesse vieler ausländischer Investoren an einer Entwicklung der SWZ-Länder. Stellen sie doch so keine ernsthafte Konkurrenz dar und bieten sie gerade wegen ihrer Unterentwicklung in vielerlei Hinsicht vorteilhafte, weil billige Standorte für die eigene Uberschuß-intensive Exportindustrie des jeweiligen Investors. Gemein ist allen Freien Wirtschaftszonen allerdings sowohl in den Industrie- als auch in den sich entwickelnden Staaten, daß es sich jeweils um ein in seinen geographischen Grenzen festgelegtes Gebiet handelt, in dem zumeist bestimmte und gesetzlich festumrissene wirtschaftliche Aktivitäten stattfinden, auf die einige ansonsten im Territorialstaat geltende gesetzliche Bestimmungen erlaubterweise keine Anwendung finden. ${ }^{29}$ Bei den Ausnahmen handelt es sich in der Regel um Befreiungen von bestimmten Steuer-, Zollund Sozialabgaben sowie eine Lockerung der Arbeits- und Sozialgesetze sowie u. U. der Verwaltungs- und Umweltgesetze. ${ }^{30}$ Es gilt aber die volle Territorialhoheit des Heimatstaats der Freizone, so daß seine Zivil-, Straf- und Verwaltungsgesetze ansonsten volle Anwendung finden.

\section{Die Entwicklungsprioritäten der chinesischen Sonderwirtschaftszonen}

Auch in den chinesischen SWZ wird vornehmlich der Export gefördert, doch unterscheiden sie sich von den sonst üblichen "Export(waren)-Verarbeitungszonen bzw. Produktionszonen" durch ihre erweiterte Zielsetzung. So kommt es den Chinesen nicht nur darauf an, vom know-how der Industrienationen im Bereich der industriellen Produktion zu lernen, sondern u. a. auch Kenntnisse auf dem Gebiet der Landwirtschaft, Nutztierzucht, Geflügelfarm, Nahrungsmittelproduktion, des Tourismus, Wohnungswesens, der Bauwirtschaft und Forschung zu erlangen. Aus diesem Grund haben die bislang vier eingerichteten SWZ z. T. unterschiedliche Entwicklungsschwerpunkte.

Die Entwicklungsprioritäten der SWZ Shenzhen (nördlich von Hongkong) liegen auf dem Gebiet der Fabrikation von Industriegütern und vor allem elektrischem Gerät (insbes. Klein-Computer), der Möbel- und Gebrauchsgüterfabrikation, dem Wohnungsbau sowie den einzelnen Zweigen der Bauwirtschaft, der Tierzucht sowie der allgemeinen Nahrungsmittelproduktion, der Förderung von Olgewinnung sowie der Petrochemie, dem Hafenbau sowie allen hiermit in Zusammenhang stehenden Industriezweigen. ${ }^{31}$

Die SWZ Zhuhai (nördlich von Macao) dient neben verschiedenen Industrieprojekten und der Olgewinnung vor allem der Förderung des Handels, des Tourismus, der Wohnungswirtschaft, der Pflanzen- und Gemüsezucht, der Fleisch- und Milchproduktion sowie anderer Landwirtschaftsprojekte. ${ }^{32}$

Shantou setzt seinen Schwerpunkt auf Exportgüterfertigung, Entwicklung der Landwirtschaft, Tierzucht, Tourismus sowie auf die Herstellung von Elektrogeräten für den

31 (4) S. $70 \mathrm{f}$. und (17) S. $155 \mathrm{f}$. 
Haushalt. Shantou ist wegen seines gut ausgebauten Hafens auch als einer der Hauptumschlagplätze für den chinesischen Außenhandel vorgesehen. ${ }^{33}$

Die SWZ Xiamen, die 1984 noch um ein Vielfaches vergrößert wurde, richtet ihr Augenmerk vor allem auf die Produktion hochwertigen elektronischen und optischen Geräts sowie auf die Herstellung anderer Präzisionsgeräte für Industrie und Forschung, daneben aber auch auf Nahrungsmittel- und Textilindustrie. Auch die Tourismusbranche soll hier besonders wegen der reichen Naturschätze gefördert werden. ${ }^{34}$

In diesen SWZ gewährt die Volksrepublik ausländischen Investoren zahlreiche Sonderkonditionen, die ihren Niederschlag in einer Vielzahl gesetzlicher Bestimmungen finden. Bevor unter 4.) die Einzelkonditionen genauer dargelegt werden, soll auf die rechtliche Verankerung der Sonderkonditionen eingegangen werden.

\section{Die gesetzliche Verankerung der Investitionsbedingungen}

Besondere Aufmerksamkeit verdient die seit 1979 fortschreitende rechtliche Verankerung der Sonderkonditionen. Zum einen, weil bislang vielen ausländischen Unternehmen ein wirtschaftliches Engagement in der Volksrepublik wegen fehlender Rechtssicherheit als zu risikoreich galt, zum anderen, weil auch die Chinesen inzwischen größten Wert darauf legen, die steigenden ausländischen Aktivitäten in ihrem Land innerhalb eines festumrissenen Rahmens zu halten. ${ }^{35}$ Dabei spielen sicherlich die leidvollen Erfahrungen aus vorrevolutionären Zeiten eine entscheidende Rolle, als die damaligen Wirtschaftszentren nicht nur von Ausländern beherrscht wurden, sondern teilweise auch unter deren Jurisdiktion standen.

Kennzeichnend für das chinesische Recht war zumindest bis Ende der 70er Jahre, daß ihm in weiten Bereichen keine Eigengeltung sondern ein nur deklaratorischer Charakter zukam. Aus der Li-Orientiertheit (Moral, Sitte und Riten) der Chinesen folgte, daß das Handeln sich nicht am geschriebenen Recht, sondern an einer in 2000 Jahren überlieferten und gef estigten Herrschafts- und Sittenordnung orientierte. ${ }^{36}$ Folglich gab es weder eine spezifische Rechtswissenschaft noch einen eigenständigen Juristenstand. Zwar kennen auch die Chinesen geschriebenes Recht (so wurden allein zwischen 1949 und 1965 ca. 1500 Gesetze und Verordnungen erlassen), ${ }^{37}$ doch kam diesem niemals die für unser Rechtssystem geltende Bedeutung zu.

Spätestens seit 1979 gibt es aber aus den genannten Gründen vor allem im Bereich des Wirtschaftsrechts Bestrebungen, dem geschriebenen Recht eine verbindliche Eigengeltung zu verleihen. So wurden neben dem Erlaß zahlreicher Wirtschaftsgesetze neue

32 (4) S. 72.

33 (4) S. 75.

34 (5) S. 76.

35 (19) S. 32.

36 (19) vgl. hierzu S. 25.

37 (19) S. 31. 
juristische Fakultäten gegründet, in denen sowohl dem alten Parteikader fehlende Rechtskenntnisse vermittelt werden sollen als auch ein neuer Juristenstand $-z$. T. unter Anleitung deutscher Rechtswissenschaftler - ausgebildet werden soll. ${ }^{38}$ Auch wurden am Volksgerichtshof Sondergerichte für Wirtschaftsstreitigkeiten eingerichtet, die über zivilrechtliche und strafrechtliche Fragen in Wirtschaftsangelegenheiten befinden. ${ }^{39}$

Die Reformen des Rechtssystems dürfen aber nicht darüber hinwegtäuschen, daß das chinesische Handeln sich weiterhin in erster Linie an den traditionellen Herrschaftsidealen ausrichtet. So betonte Deng Xiaoping in einer Rede vor der Nationalen Konferenz über Wissenschaft und Technik am 7. März 1985,40 daß oberster Orientierungsrahmen für die Modernisierung Chinas die Fünf Gebote (Anstand, Höflichkeit, Hygiene, Zucht und Ordnung, gute Moral), die Vier Tugenden (korrekte Geisteshaltung, höfliche Sprache, gesittetes Verhalten, schöne Umwelt) und die Drei Lieben (Liebe zum Vaterland, Liebe zum Sozialismus und Liebe zur Partei) seien.

Insbesondere die Fünf Gebote, die Vier Tugenden sowie die Liebe zum Vaterland stellen nicht bloße Programmsätze sondern seit langem gefestigte und internalisierte Handlungsideale mit Normcharakter dar. Normcharakter haben allerdings auch die beiden Lieben neueren Datums, die Liebe zum Sozialismus und die Liebe zur Partei, wie die zahlreichen pol. Prozesse der Vergangenheit immer wieder gezeigt haben.

Es wäre daher falsch, aus der momentanen Emsigkeit des chinesischen Gesetzgebers die Schlußfolgerung zu ziehen, die Volksrepublik befände sich auf dem Weg zu einem Rechtssystem europäischer Prägung. Dies hieße, sowohl die chinesische Rechtstradition als auch den chinesischen Sozialismus in seiner Eigenart zu verkennen.

\section{Die wichtigsten Sonderkonditionen in den chinesischen Sonderwirtschaftszonen}

\subsection{Steuern}

Die wichtigsten Gesetze:

- Income Tax Law Concerning Joint Ventures Using Chinese and Foreign Investment vom 10. 9. $1980^{41,42}$

- Individual Income Tax Law vom 10.9.198043

- Income Tax Law Concerning Foreign Enterprises vom 13. 12. 198144,45

- Consolidated Industrial and Commercial Tax Regulations vom 13.9. $1958^{46}$

38 Beijing Rundschau Heft 18 vom 7. 5. 1985, S. 23 f.

39 Beijing Rundschau Heft 11 vom 19. 3. 1985, S. 24.

40 Beijing Rundschau, Heft 11 vom 19. 3. 1985.

41 Quelle: (4) S. 427.

42 Dieses Gesetz wird erläutert von F. Münzel in Recht der Internationalen Wirtschaft, Heft 1 1981, S. 24 ff.

43 Quelle: (4) S. $433 \mathrm{ff}$.

44 Quelle: (4) S. 437 ff.

45 Das Gesetz wird erläutert von Sabine Hug in Recht der Internationalen Wirtschaft, Heft 11 1982, S. 808 ff.

46 Quelle: (4) S. 415 ff. 
In den SWZ beträgt der Einkommenssteuersatz ${ }^{47}$ für ausländische Unternehmen sowie chinesisch-ausländische Gemeinschaftsunternehmen nur $15 \%$, wobei gewisse Unternehmen in den ersten Jahren ihrer wirtschaftlichen Betätigung regelmäßig eine weitere Steuerermäßigung zwischen 20 und $50 \%$ bzw. sogar eine völlige Steuerbefreiung für die ersten profitbringenden Jahre erhalten können..$^{48}$ Der gesamte Steuersatz in der Volksrepublik beträgt $33 \%$ und setzt sich aus einer $30 \%$ igen nationalen Steuer zuzüglich einer lokalen Steuer in Höhe von $10 \%$ der nationalen Steuer zusammen. ${ }^{49}$

Eine weitere wichtige Steuer ist die Industrie- und Handelssteuer. ${ }^{50}$ Die gesetzlichen Bestimmungen stammen aus den 50er Jahren und sind größtenteils veraltet. Die Besteuerungsquote variiert zwischen 1,5\% für gewisse Baumwollprodukte und $69 \%$ für Zigaretten. ${ }^{51}$ Allerdings werden die von den ausländischen Unternehmen importierten Produktionsmittel nicht besteuert. Für alle Einfuhren kann die Steuer ermäßigt oder erlassen werden. ${ }^{52}$ Gleiches gilt für die für den Export produzierten Waren.

\subsection{Zölle}

Für die Erhebung von Im- und Exportzöllen gibt es eine ganze Reihe von Gesetzen, ministeriellen Verordnungen und Einzelweisungen der lokalen Verwaltungen. ${ }^{53}$

In der Regel werden in China sowohl Import- als auch Exportzölle erhoben. Letztere erst seit dem 1. 6. 1982. ${ }^{54}$ Bei den Importzöllen wird zwischen Mindest- und Normaltarifen unterschieden. Erstere gelten für Importe aus Ländern mit denen die Volksrepublik ein besonderes Handelsabkommen hat. Luxusgüter können bis zu $400 \%$ verzollt werden. ${ }^{55}$

Ausländische Unternehmen, die in der SWZ tätig sind, können Ausrüstungsgegenstände, Rohstoffe sowie andere zur Produktion benötigte Gegenstände und Materialien zollfrei einführen. Zollfrei ist auch die Ausfuhr der in den SWZ für den Export hergestellten Waren. ${ }^{56}$ Konsumgüter, die in den SWZ verkauft werden sollen, sind mit Ausnahme von Tabakwaren, Alkohol, Getränken sowie hochwertigen Kosmetika von Einfuhrzöllen befreit. ${ }^{57}$ Werden sie jedoch aus den SWZ in die Volksrepublik eingeführt, fallen hohe Zölle an.

In der VR China wird nicht zwischen den Begriffen Einkommenssteuer und Körperschaftssteuer unterschieden, sondern Steuern auf das Einkommen natürlicher sowie juristischer Personen regelmäßig als Einkommenssteuer bezeichnet.

48 (4) S. 68.

49 (9) S. 18.

50 Quelle: (4) S. 415 ff.

$51 \mathrm{Vgl}$. Tabelle für Besteuerungsquoten (4) S. $416 \mathrm{ff}$.

52 In der Regel besteht die "Einfuhrsteuer" aus der Industrie- und Handelssteuer sowie aus den Zöllen. Beide werden fällig, aber in der Regel nicht als einheitliche Steuer erhoben.

53 (4) S. $455 \mathrm{ff}$.

54 Vgl. (10) S. 451.

55 Ebd.

56 (4) S. 69.

57 Ebd. 


\subsection{Devisenbestimmungen}

Die wichtigsten Gesetze:

- Regulations on Special Economic Zones in Guandong Province vom 26. 8. $1980^{58}$ sowie zahlreiche andere Devisen- und Devisenkontrollbestimmungen. ${ }^{59}$

Die Währung der Volksrepublik heißt Renminbi (RMB). Die Zahlungseinheit bildet der Yuan. In den SWZ werden z. T. auch Devisen - vor allem der Hongkong Dollar sowie der US Dollar - als Zahlungsmittel gehandelt und akzeptiert.

Unternehmensprofite, Löhne und Gehälter sowie andere rechtmäßige Verdienste dürfen gem. Art. 15 der o. a. Bestimmungen für die SWZ nach Zahlung von Steuern und anderen vorgeschriebenen Abgaben über die Bank von China ins Ausland transferiert werden. Dieser Grundsatz findet seinen Niederschlag auch in anderen Gesetzen, wie in Art. 7 des Gesetzes für chinesisch-ausländische Gemeinschaftsunternehmen (Law on Joint-Ventures Using Chinese and Foreign Investment vom 8. 7. 1979). ${ }^{60}$

\subsection{Vermietung und Verpachtung}

Die wichtigsten Gesetze

- Interim Provisions of the Shenzhen Special Economic Zone for Land Management vom 24. 12. $1981^{61}$ (Interimsregeln)

- Regulations of the Shenzhen Special Economic Zone Concerning the Management of Commodity House Property vom 23. 1. $1984^{62}$

Diese wichtigen Regeln für die Vermietung und Verpachtung von Grund und Boden in der SWZ von Shenzhen werden ebenso in den anderen SWZ angewandt.

Ausländische Unternehmen können Grund und Boden zu günstigen Konditionen mieten und pachten. ${ }^{63}$ Gemäß Art. 5 Interimsregeln dürfen Nutzungsrechte, aber keine Eigentumsrechte an Grund und Boden vergeben werden. Eine andersartige Regelung würde auch den Artikeln 6, 8, und 10 der Verfassung vom 4. 12. $1982^{64}$ widersprechen, die Privateigentum an Grund und Boden ausschließen. Dies besagt aber nicht, daß - anders als im deutschen Recht (vgl. §§ 93, 94 BGB) - kein getrenntes Eigentum an den mit Grund und Boden fest und auf Dauer verbundenen Gebäuden und Einrichtungsgegenständen möglich ist.

Gemäß Art. 5 der Regulations of the Shenzhen Special Economic Zone Concerning the Management of Commodity House Property vom 23. 1. 1984 wird der Unternehmer, der eine Industrieanlage, ein Handelsgebäude, Warenhaus, einen Parkplatz oder ein

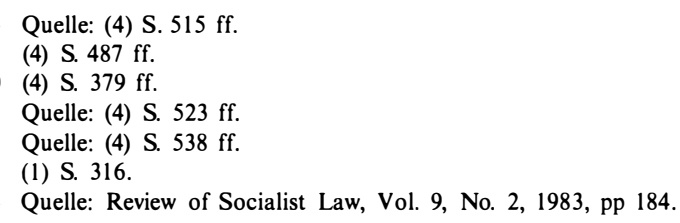


anderweitiges Gebäude - auch Privathäuser - aus eigenen Mitteln errichtet, Eigentümer dieses Bauwerks. Bei Gemeinschaftsprojekten werden die Beteiligten in Höhe ihrer jeweiligen Kapitalbeteiligung Eigentümer. Beim Verkauf geht das Eigentum an den Gebäuden - unabhängig vom staatlichen Eigentum an Grund und Boden - auf den Käufer über.

Gemäß Art. 15 der Interimsregeln können Miet- bzw. Pachtverträge für Grund und Boden je nach Art und Weise der Nutzung für 20 (z. B. für den Besitzer eines Restaurants) bis längstens 50 Jahre (z. B. für technologische, wissenschaftliche oder medizinische Einrichtungen) abgeschlossen werden.

Der jährliche Miet- bzw. Pachtzins für Grund und Boden beträgt z. B. für Industrieanlagen zwischen 10-30 Yuan pro $\mathrm{m}^{2}$ und für Tourismuseinrichtungen zwischen 60-100 Yuan pro $\mathrm{m}^{2}$ (Art. 16 Interimsregeln). Der Pachtzins für Landwirtschaftsprojekte unterliegt einer besonderen Vereinbarung (Art. 16 Interimsregeln).

Die Interimsregeln sehen desweiteren alle 3 Jahre eine Anpassung der Miet- und Pachtzinsen an die allgemeinen Preissteigerungen vor (Art. 16) sowie Vergünstigungen und Befreiungen für bestimmte Projekte (Art. 17).

\subsection{Arbeit und Löhne}

Wichtigste Gesetze:

- Regulations on Special Economic Zones in Guangdong Province vom 26. 8. 198065 (Regeln für die SWZ)

- Interim Provisions for Labour and Wage Management in Enterprises in the Special Economic Zones in Guangdong Province vom 24. 12. $1981^{66}$ (Interimsregeln)

Eine der vielleicht gravierendsten Veränderungen ist im Bereich Arbeit und Löhne zu verzeichnen. War bislang den Chinesen ihr Arbeitsplatz sicher und galt hinsichtlich der Entlöhnung das Prinzip der "eisernen Reisschale" (alle essen aus dem gleichen Reistopf), so herrscht nun in den SWZ z. T. aber auch bereits im restlichen China, das Prinzip des Heuerns und Feuerns sowie ein an der individuellen Leistung orientiertes Prämiensystem.

Gemäß Art. 19 o. a. Regeln für die SWZ sowie Art. 2 der o. a. Interimsregeln dürfen Unternehmer Arbeitnehmer vor der Einstellung testen und schließen sie mit diesen bislang in China unübliche - Arbeitsverträge ab, die jedoch zumeist vom zuständigen Arbeitsamt genehmigt werden müssen.

Gemäß Art. 20 der Regeln für die SWZ dürfen Arbeitnehmer im Einklang mit den Bestimmungen des Arbeitsvertrags entlassen werden und dies nicht nur bei vertragswidrigem Verhalten sondern gemäß Art. 16 der Interimsregeln auch unter bestimmten Voraussetzungen bei notwendigen Rationalisierungsmaßnahmen. Gemäß Art. 2 der Regeln 
für die SWZ sowie Art. 8, 9 der Interimsregeln können die Unternehmen das Gehaltssystem (Grundgehalt plus je nach Leistung variierender Prämie) selbst bestimmen.

\subsection{Ein- und Ausreisebestimmungen}

Wichtigste Gesetze:

- Interim Provisions of the Special Economic Zones in Guangdong Province for the Control of Personnel Entering and Leaving China ${ }^{67}$

Bislang mußten Visa für die Einreise in SWZ auf dem langen Dienstweg über Beijing erteilt werden. Da diese Regelung sich aber als äußerst ineffektiv erwies und zahlreiche Ausländer vergraute, zumal die Grenzen zwischen Hongkong und Shenzhen bereits im Jahre 1982 täglich ca. 12000 mal überquert wurde, ${ }^{68}$ mußte hier Abhilfe geschaffen werden. Die chinesischen Auslandsvertretungen dürfen nun selbst Einreisegenehmigungen erteilen und Dauerreisende können ein Dauervisum bis zu einem Jahr erhalten. In einigen Fällen dürfen selbst die Zollbehörden an den Grenzen Visa ausstellen. ${ }^{69}$

\subsection{Sonstiges}

Weitere wichtige Rahmenbedingungen für ausländische Investoren sind die "Interims Provisions for the Registration and Administration of Enterprises in the Special Economic Zones in Guangdong Province" vom 24. 12. 1981, ${ }^{70} "$ Rules for the Implementation of the Registration and Administration of Enterprises in the Shenzhen Special Economic Zones" vom 9. 2. 1984, ${ }^{71}$ "Interim Provisions on Technology Imports in Shenzhen Special Economic Zone" vom 8. 2. $1984^{72}$ sowie vor allem die "Provisions of the Shenzhen Special Economic Zone for Foreign Economic Contracts" vom 7. 2. 1984. ${ }^{73}$ Letzteres Gesetz ist das Außenwirtschaftsvertragsgesetz der SWZ von Shenzhen und gleicht sowohl in Form und Inhalt dem neuen Gesetz der VR China über Außenwirtschaftsverträge vom 21. 3. $1985,{ }^{74}$ das am 1. 7. 1985 in Kraft trat. Von weiterer Bedeutung sind das Gesetz für chinesisch-ausländische Gemeinschaftsunternehmen vom 8. 7. 1979, ${ }^{75}$ das Wirtschaftsvertragsgesetz vom 13. 12. 1981, ${ }^{76}$ die Regeln zur Beilegung von Streitigkeiten über Wirtschaftsverträge ${ }^{77}$ sowie das chinesische Warenzeichenrecht vom 23. 8.

67 Quelle: (4) S. $519 \mathrm{f}$.

68 The Journal of Commerce vom 25. 8. 1982.

69 (4) S. 66.

70 Quelle: (4) S. $517 \mathrm{ff}$.

71 Quelle: (4) S. $525 \mathrm{ff}$.

72 Quelle: (4) S. $529 \mathrm{ff}$.

73 Quelle: (4) S. 523 f.

74 Quelle: Renmin-ribao (Volkszeitung, Beijing) vom 22. 3. 1985.

75 Quelle: (4) S. 379 ff. "Law on Joint-Ventures Using Chinese and Foreign Investment".

76 Quelle: (4) S. 574 ff.

77 Quelle: (4) S. $584 \mathrm{ff}$. 
$1982^{78}$ und das Patentgesetz vom 12. 3. $1984^{78 a}$ mit seinen Ausführungsbestimmungen (Entwurf) vom 19. 1. 1985. ${ }^{78 \mathrm{~b}}$

Für deutsche Investoren haben zudem folgende bilateralen Abkommen erhebliche Bedeutung: Das "Abkommen zwischen der Regierung der Bundesrepublik Deutschland und der Regierung der Volksrepublik China über wirtschaftliche Zusammenarbeit" vom 24. Oktober $1979,{ }^{79}$ dessen Gültigkeitsdauer dieses Jahr bis zum 31. Dezember 1995 verlängert wurde ${ }^{80}$ das "Abkommen zwischen der Bundesrepublik Deutschland und der Volksrepublik China über die Förderung und den gegenseitigen Schutz von Kapitalanlagen vom 7. Oktober 1983, ${ }^{81}$ das am 20. Dezember 1984 in Kraft getreten ist, sowie das "Abkommen zur Vermeidung der Doppelbesteuerung auf dem Gebiet der Steuern von Einkommen und Vermögen", das vor kurzem unterzeichnet wurde, aber noch nicht in Kraft getreten ist. ${ }^{82}$

\section{Schlußfolgerungen}

Nach Darstellung der Wesenszüge der chinesischen Reformpolitik im Bereich der Wirtschaft unter besonderer Berücksichtigung der Sonderwirtschaftszonen heißt es nun, den Kreis zu schließen und an die bereits zu Anfang aufgeworfene Frage anzuknüpfen, nämlich: Befindet sich die VR China auf dem Weg zu einem kapitalistischen System? Betrachtet man die Reformen losgelöst von ihren "gesetzlichen" Rahmenbedingungen und ihrer Eingebundenheit in das chinesische Gesellschaftssystem, so möchte man die Frage bejahen. Sind doch die Begrenzung staatlicher Einflußnahme auf Wirtschaftsplanung und Wirtschaftstätigkeit, die Zulassung privater Unternehmen sowie die Orientierung von Produktion und Markt an Angebot und Nachfrage, das Prinzip des Heuerns und Feuerns sowie ein an der individuellen Leistung ausgerichtetes Entlöhnungssystem u. a. Wesensmerkmale eines kapitalistischen Wirtschaftssystems.

Eine solch losgelöste Betrachtungsweise führt aber meines Erachtens zu falschen Schlußfolgerungen. Ich möchte daher im folgenden und abschließend fünf Thesen vortragen, die mich dazu veranlassen, oben aufgeworfene Frage zu verneinen:

(1) Erstens, die Wirtschaftsreformen sind die pragmatische Konsequenz aus den negativen Erfahrungen mit der zentralistischen Planwirtschaft sowie aus eigenen Versäumnissen.

Wie schon erwähnt, zeigte sich das kollektivistische Landwirtschaftssystem als ungeeignet, den bestehenden sowie den steigenden Bedarf an Grundnahrungsmitteln zu befriedi-

78 Quelle: (4) S. $559 \mathrm{ff}$.

78a Quelle: (4) S. 567 ff.

78b Quelle: Beijing Rundschau, Heft 7/8 vom 12. 5. 1985.

79 Quelle: Bundesgesetzblatt Teil II 1979, S. 1209 ff.

80 Bundesgesetzblatt Teil II 1985, S. 1043.

81 Quelle: Bundesgesetzblatt Teil II 1985, S. $30 \mathrm{ff}$.

82 Süddeutsche Zeitung vom 7. 6. 1985. 
gen. Der Industriebereich ist größtenteils rückständig und arbeitet ineffizient und die unternehmerische Entscheidungsfreiheit ist (bzw. war) infolge einer fast unübersehbaren Anzahl zentralstaatlicher Eingriffsbefugnisse gelähmt. ${ }^{83}$ Gewinne waren sofort und in voller Höhe an den Staat abzuführen, während Verluste ineffizienter Betriebe in voller Höhe gedeckt wurden. ${ }^{84}$ In der Unternehmensverwaltung sowie unter Arbeitern und Angestellten war wegen fehlender Leistungsanreize der allgemeine Schlendrian verbreitet. Das egalitäre Lohnsystem lähmte - trotz seines seinerzeit durchaus berechtigten Anlasses - auf Dauer die Initiativkraft der Beschäftigten.$^{85}$ Die zentralstaatliche Wirtschaftsplanung vermochte weder das steigende Konsumbedürfnis der Bevölkerung zu befriedigen noch die steigende Arbeitslosigkeit besonders unter den Jugendlichen ${ }^{86} \mathrm{zu}$ lösen.

(2) Zweitens, die meisten Wirtschaftsreformen haben vorerst nur Modell- und Versuchscharakter.

Bevor die VR China zu den umfangreichen Reformmaßnahmen griff, informierten sich chinesische Wirtschaftsfachleute umfassend über anderweitig herrschende Marktmechanismen. Das Studium ausländischer Marktkenntnisse, moderner Technologien und Managementmethoden in den industrialisierten Staaten ${ }^{87}$ sowie die genaue Beobachtung der Arbeitsweise verschiedener Freier Wirtschaftszonen in sich entwickelnden Län$\operatorname{dern}^{88}$ führte bislang zu einer lediglich punktuellen Erneuerung der chinesischen Wirtschaft. So werden vorerst nur eigens ausgewählte Betriebe und bestimmte Industriezweige reformiert, um anhand der hier gewonnenen Erfahrungen anschließend endgültige Entscheidungen treffen zu können.

Ähnliches gilt für die SWZ. Von ihnen erhofft man sich langfristig positive Auswirkungen auf die wirtschaftliche Entwicklung des chinesischen Hinterlandes. Vorerst aber dienen sie als Labor zum Ausprobieren neuer Ideen und Maßnahmen. Neue Erfahrungen sollen erst dann für die gesamte Volksrepublik übernommen werden, wenn sie sich als effektiv und nützlich erwiesen haben und das "restliche" China für eine Ubernahme "reif" genug ist. ${ }^{89}$ Mit aus diesem Grund sind die SWZ vom "restlichen" China auch hermetisch abgekapselt. ${ }^{90}$ So trennt bereits ein $84,6 \mathrm{~km}$ langer und stark kontrollierter Grenzzaun mit nur wenigen Kontrollübergängen die SWZ Shenzhen vom chinesischen Hinterland. ${ }^{91}$

(3) Drittens, die Wirtschaftsreformen sind eingebunden in ein sozialistisches Gesellschaftssystem.

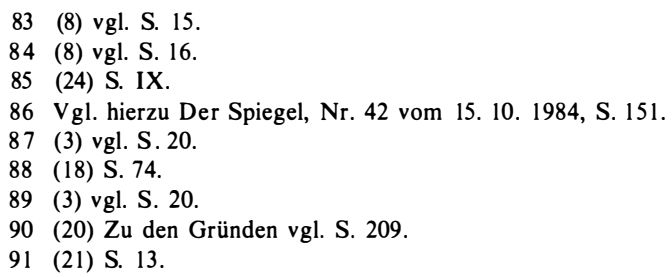


Die Einführung effizienter Marktmechanismen muß nicht notwendigerweise eine Veränderung der gesellschaftspolitischen Grundstruktur nach sich ziehen. Zum einen haben sich zahlreiche ökonomische Gesetze und Prinzipien als grenz- und gesellschaftsüberschreitend erwiesen, ${ }^{92}$ zum anderen bestimmen die gesellschaftspolitischen Rahmenbedingungen der Volksrepublik die Stoßrichtung der Reformen.

Die chinesische Verfassung vom 4. Dezember $1982^{93}$ läßt trotz ihres nur deklaratorischen Charakters und ihrer Wirkung eines Verhaltenskodex (vgl. Präambel) keinen Zweifel daran, daß die VR China ein sozialistischer Staat ist. Das Bekenntnis zum Sozialismus ist und bleibt das Grundprinzip allen Handelns (vgl. Präambel, Art. 1 und Art. 3). Gemäß Art. 6, 9 und 10 verbleiben Grund und Boden sowie die wesentlichen Produktionsmittel im Gemeineigentum. Für den Grund und Boden gilt dies auch in den SWZ. ${ }^{94}$ Gemäß Art. 12 ist das sozialistische Gemeineigentum heilig und unverletzlich, und die staatliche Wirtschaftsplanung hat sich gemäß Art. 15 am Grundsatz des sozialistischen Gemeineigentums auszurichten.

Gemäß Art. 22 haben der gesamte Kulturbereich sowie die Massenmedien Volk und Sozialismus zu dienen, und die Volkserziehung muß sich an den Prinzipien des Patriotismus, Kollektivismus, Internationalismus und Kommunismus sowie am dialektischen und historischen Materialismus orientieren und soll kapitalistischen, feudalen und anderen dekadenten Ideologien entgegenwirken (Art. 24).

Diese Verfassungsgrundsätze finden ihren Niederschlag auch in den Einzelgesetzen. So betonen verschiedene Einzelgesetze das Prinzip des sozialistischen Gemeineigentums an Grund und Boden. In zahlreichen Reden der chinesischen Führung wird immer wieder die sozialistische Ausrichtung der Reformen in den Vordergrund gerückt und vehement Kritik an manchen "kapitalistischen" Auswüchsen dieser Erneuerungen geübt und die Bevölkerung vor den Konsequenzen gewarnt.95

(4) Viertens, die chinesischen Wirtschaftsreformen finden Parallelen in anderen sozialistischen Staaten.

Auch andere sozialistische Staaten haben zur Ankurbelung ihrer Wirtschaft auf grenzüberschreitende Marktmechanismen kapitalistischer Staaten zurückgegriffen, ohne daß dies als ein Signal für eine Abkehr vom Kommunismus gedeutet wurde. So gibt es insbesondere in Jugoslawien und Ungarn kommunistisch-kapitalistische Gemeinschaftsunternehmen. Gerade in Ungarn ist freies Unternehmertum in einem festumrissenen) Rahmen erlaubt und erwünscht, und mittlerweile gibt es sogar Aktiengesellschaften und somit Börsenspekulanten.

(5) Fünftens, die chinesischen Wirtschaftsreformen finden ihre ideologische Begründung u. a. in Lenins Thesen zum Staatskapitalismus.

92 (18) vgl. S. 73.

93 Quelle: Review of Socialist Law, Vol. 9, No. 2, 1983, pp 184.

$94 \mathrm{Vgl}$. Kapitel IV. 4.4 sowie (1) S. $316 \mathrm{f}$.

95 Vgl. stellvertretend Rede Deng Xiaoping's vom 22. 10. 1984, abgedruckt in Beijing Rundschau Heft 7/8 vom 12. 5. 1985. 
In letzter Zeit beruft sich die chinesische Führung zur ideologischen Untermauerung ihrer Reformen häufig auf Lenin. ${ }^{96}$ Bereits Anfang der 20er Jahre hatte Lenin die Beteiligung des kapitalistischen Auslands am Modernisierungsprogramm der Sowjetunion befürwortet. Um die Produktmenge zu steigern, schlug Lenin ein "Konzessionssystem "97 vor. Danach sollten "Kapitalisten" Produktionsmittel (Betriebe, Bergwerke, Rohstoffe, Maschinen etc.) zu günstigen Pachtzinsen und einen guten Anteil am Profit erhalten, den sie auch transferieren durften. Lenin versprach sich hiervon im Gegenzug eine umfassende Erneuerung der verpachteten Industrieunternehmen sowie einen raschen Anstieg der Produktion. ${ }^{98}$

Darüberhinaus propagierte Lenin die Existenz einer privaten Kleinwirtschaft, um den chronischen Mangel u. a. an Grundnahrungsmitteln, Brennmaterialien, Rohstoffen sowie Transportmitteln beseitigen zu können. ${ }^{99}$ Bauern sollten das Recht bekommen, ihre Uberschüsse nach Abgabe einer Naturalsteuer frei auszutauschen. ${ }^{100}$

Lenin machte keinen Hehl daraus, daß diese "kapitalistischen Methoden "101 auch Gefahren für das sozialistische System in sich bergen. Grundsätzlich war er aber der Uberzeugung, daß sowohl pragmatische als auch ideologische Gründe für sie sprachen: Solange der Kapitalismus sich in einem Staat entfalte, in dem die Schlüsselgewalt in den Händen des Volkes und nicht der Kapitalisten liege, in dem Grund und Boden sowie wesentliche Produktionsmittel im Gemeineigentum ständen und kapitalistische Aktivitäten vom sozialistischen Staat kontrolliert und registriert würden, ${ }^{102}$ bedeute die Freiheit des Kapitalismus gleichzeitig eine neue Form des Kapitalismus, nämlich die des Staatskapitalismus. ${ }^{103}$

Diese Leninschen Grundgedanken zum Staatskapitalismus macht sich zunehmend auch die chinesische Führung zu eigen. Einerseits, um die Wirtschaftsreformen gegenüber Kritikern aus den eigenen Reihen auch ideologisch rechtfertigen zu können, andererseits, um auch gegenüber dem Ausland keinen Zweif el an der sozialistischen Grundausrichtung der VR China aufkommen zu lassen. ${ }^{104}$

(18) vgl. S. 74.

(13) vgl. S. $650 \mathrm{ff}$.

(13) vgl. S. $650 \mathrm{ff}$.

(13) S. 649.

(14) S. 675.

(13) S. 651.

(13) vgl. S. $651 \mathrm{f}$.

(14) vgl. S. 675.

104 Vgl. zum chinesischen Sozialismus Karl Kränzle, Das Ende der eisernen Reisschale, in der Süddeutschen Zeitung vom 7. 11. 1984 sowie derselbe, Mit Konfuzius angereicherter Sozialismus, in der Süddeutschen Zeitung vom 30. 4. 1985. 


\section{Literaturverzeichnis:}

(1) Barrett, Jill: What's New in China's New Constitution in Review of Socialist Law, Vol. 9, No. 4, 1983, pp 305

(2) Chevrier, Yves: Les politiques économiques de la démaơisation (1977-1982), in Revue d'Etudes Comparatives Est-Ouest, Vol. XIV, No. 3, Sept. 1983, pp 5

(3) Chen Qiwei: Warum öffnet sich China?, in Beijing Rundschau Heft 13, 1985

(4) The China Investment Guide 1984/85, China International Economic Consultants Inc., Hongkong 1984

(5) China, Socialist Economic Development, A World Bank Country Study, Vol. I, 1984

(6) Deng Xiaoping: Rede am 7. März 1985 vor der nationalen Konferenz über Wissenschaft und Technik, abgedruckt in Beijing Rundschau Heft 11, 1985

(7) Grubel Herbert G.: Free Economic Zones: Good or Bad?, in Außenwirtschaft, Schweizerische Zeitschrift für internationale Wirtschaftsbeziehungen, 39. Jahrg., Heft I/II Mai 1984, S. 43 ff.

(8) Huang Xiang: Rede über die Reform des chinesischen Wirtschaftssystems auf dem Symposium "China und die Zukunft" am 8. 5. 1984 in Beijing, abgedruckt in Beijing Rundschau Heft 20, 1985

(9) Jao, Y. C.: Recent Developments in China's Tax System, in Bulletin for International Fiscal Documentation, Vol. 35, 1981, pp 18

(10) Jehle, Eugen: The Tax System of the People's Republic of China - A Short Survey, in Bulletin for International Fiscal Documentation, Vol. 36, 1982, pp 447

(11) Kummer, Heinz-Günter: Die im Schatten als Vorbild, in Die Zeit vom 12. 10. 1984

(12) Laaksonen, Oiva: The Management and Power Structure of Chinese Enterprises during and after the Cultural Revolution, in Organization Studies, No. 5, Issue I 1984, pp 1

(13) Lenin, Wladimir Iljitsch: Uber die Naturalsteuer, Referat abgedruckt in N. Lenin: Der Kampf um die Soziale Revolution, 1925, S. 638 ff.

(14) Die Taktik der Kommunistischen Partei Rußlands, Rede vom 5. 7. 1921. abgedruckt ebd. S. 662 ff.

(15) Li Honglin: Offnungspolitik - ein Schlüssel für den Sozialismus - in Beijing Rundschau Heft 13, 1985

(16) Nishitateno, Sonoko: China's Special Economic Zones: experimental units for economic reform, in The International and Comparative Law Quarterly, Vol. 70, Part 1, January 1983, pp 175

(17) Roy, Michel: Shenzhen: Une zone économique spéciale en chine populaire, in Revue d'Etudes Comparatives Est-Ouest, Vol. XIV No. 3, Sept. 1983, pp 153

(18) Sit, Victor F. S.: The Special Economic Zones of China: A New Type of Export Processing Zone?, in The Developing Economics, Vol. XXIII, No. I, March 1985, pp 65

(19) Weggel, Oskar: Das chinesische Rechtssystem, in Garms (Hrsg.): Wirtschaftspartner China 1981/82, 1981, S. $25 \mathrm{ff}$.

(20) Der institutionelle Rahmen der internationalen wirtschaftlichen Beziehungen der VR China, in Chinas neuer Weg zum Recht, 1982, S. 191 ff.

(21) Williams, Tim and Brilliant, Robin: Shenzhen Status Report, in The China Business Review, March-April 1984, Vol. 11, No. 2, pp 10

(22) Wulf, Luc de: Wirtschaftsreform in China, in Finanzierung \& Entwicklung, Heft 11, März 1985, S. 8 ff.

(23) Zagoria, Donald: China's Quiet Revolution, in Foreign Affairs, spring 1984, pp 879

(24) Zhao Ziyang: Bericht über die Tätigkeit der Regierung zur 3. Tagung des VI. Nationalen Volkskongresses am 27. 3. 1985, abgedruckt in Beijing Rundschau Heft 16, 1985 
account that the choice of which sectors of society are to bear the burdens entailed by adjustment programmes is a decision of their own governments. Protests ought therefore to be directed against governments and not against the IMF.

\title{
Heading Towards a Second Natural Rubber Agreement?
}

\author{
By Andreas A. B. Hoffmann
}

The International Natural Rubber Agreement of 1979, a commodity price stabilization accord between natural rubber exporting and importing nations, will be renegotiated in 1986. The two groups are divided over the question, whether to include production costs into the formula for calculating the reference price.

Despite diverging views on most issues, producers and consumers agree that chances for concluding a second pact are rather high, compared to futile efforts to achieve working agreements for other raw materials.

It is argued, however, that industrialized rubber importing countries fared better with the present agreement than did the developing exporters, who start to feel the financial burden of maintaining a 380,000 tons buffer stock.

\section{The Chinese Economic Reforms since 1978 with Particular Regard to the Special Econo- mic Zones}

\section{By Martin Klingst}

Since 1978 the People's Republic of China (PRC) has undertaken vast agrarian and industrial reforms. These reforms were distinctly welcomed in capitalist countries as well as harshly criticized by Chinese opponents and various foreign communist movements and parties.

This article, therefore, starts with a question: Is the PRC returning back to capitalism? In the following it deals with the different legal, economic and political aspects of the economic reforms and focuses on the establishment of the so-called "Special Economic Zones", because it was mainly these that gave rise to the above mentioned discussion. Special Economic Zones are zones of free enterprise - mostly export-processing-zones and are to be found all over the world. But especially in the so-called developing countries they provide many favorable conditions for local and foreign investors.

After describing and analyzing the various general aspects of Special Economic Zones and details about the Chinese Special Economic Zones the article ends with the conclusion that the PRC is changing but will nevertheless remain a socialist country, because: 
firstly, the economic reforms are in part merely the pragmatic lessons learned from past failures; secondly, most of the economic reforms only serve as temporary models; thirdly, the Chinese economic reforms remain integrated into a basically socialist system; fourthly, other communist countries have already established and carried out similar reforms and, fifthly, the reforms find their theoretical background in Lenin's theory of state-capitalism.

\section{International Disputes in Africa}

\section{By Maria Magdalena Kenig}

The paper discusses problems of the peaceful settlement of international disputes in Africa.

About sixty disputes are discussed which arose in the years 1945-1985. This study attempts to establish the rôle and contribution of African states to maintenance of security in Africa, as well as to define the nature of the process from the point of view of the creation of norms of regional law in Africa. One of the hypotheses put forward states that there is a clear relationship between the processes for the settlement of international disputes in Africa and the overall development of contemporary international relations. Studies of importance for a discussion of the settlement of international disputes in Africa have been attempted at two levels, i. e. at the level of relevant African positive international law and at the level of the practical measures taken by states.

The survey of problems of the settlement of international disputes in Africa permits the conclusions that African states have created institutions, principles and norms of a regional legal order. This activity comprises not only modifications relating to substantive international law but also to its forms and structures. The custom developed in this field is accepted to the African community.

As a rule, African states have adopted diplomatic procedures, whose characteristics and nature are difficult to define. They do, however, possibilities of achieving far-reaching compromises and political arrangements on controversial issues, which clearly contributes to keeping these processes of settlement within a strictly African framework.

It is not easy to know the legal nature of the ad-hoc procedures utilised by African states. Despite a reluctance to adopt formal rules, there are some tendencies to establish principles which govern this area of international relations. These principles are based on African tradition in which African diplomacy is rooted. 